\title{
Revisión sobre el manejo de atresia de conducto auditivo externo mediante el uso de dispositivos auditivos implantables osteointegrados: A propósito de un caso exitoso con un implante Bonebridge ${ }^{\circledR}$ \\ Review of the management of external auditory canal atresia using Osseo integrated implantable hearing devices: Report of a successful case with Bonebridge ${ }^{\circledR}$ implant
}

\author{
David Sanhueza C², Patricia Esquivel C1, Claudia Dentone S1.
}

\begin{abstract}
RESUMEN
La atresia de conducto auditivo externo tiene una incidencia de 1 por 1.000 ó 2.000 nacidos vivos, predominantemente unilateral. Su manejo actual es controversial, en especial la cirugía de reconstrucción. En el último tiempo se han desarrollado dispositivos osteointegrados, que han revolucionado el tratamiento y cuestionado la indicación de cirugía.

El liderazgo por un tiempo importante lo ha tenido el dispositivo tipo BAHA, pero que ha tenido problemas en el largo plazo, principalmente en relación con el tornillo del implante. En respuesta a estos problemas, se ha diseñado un nuevo implante osteointegrado de nombre Bonebridge ${ }^{\circledR}$, con beneficios frente a su antecesor.

Como en nuestro medio se tiene poca experiencia en este dispositivo se presenta una revisión a propósito de un caso clínico exitoso. El caso corresponde a un paciente de 18 años con hipoacusia de conducción máxima bilateral congénita, con diagnóstico de atresia de conducto auditivo externo bilateral, con inadecuada ganancia auditiva del cintillo óseo tradicional.

Tanto la revisión bibliográfica como el caso clínico nos permiten aseverar que Bonebridge ${ }^{\circledR}$ corresponde a tratamiento adecuado en el manejo de la atresia del conducto auditivo externo, con ciertos beneficios sobre otros dispositivos y la cirugía.

Palabras clave: Atresia de conducto auditivo externo, Bonebrigde ${ }^{\circledR}$.
\end{abstract}

\begin{abstract}
The external auditory canal atresia has an incidence of 1 per 1000 or 2000 live births predominantly unilateral. Its management is controversial, especially with the decision of reconstructive surgery. Lately, have developed osseo integrated bone conduction
\end{abstract}

Médicos Servicio ORL, Hospital Barros Luco Trudeau.

2 Médico. Universidad de Chile. 
devices, which have come to revolutionize the treatment and questioning the indication for surgery.

The leadership for a long time its has had it the BAHA device type, but has several problems in long-term, mainly in relation to the screw used in the implant. In response to this problems, was designed a new osseo integrated implant named Bonebridge ${ }^{\circledR}$, showing benefits over its predecessor.

As in our setting there is little experience with this device, we present a review about a successful clinical case. The case is a patient of 18 years with a maximal bilateral hearing loss caused by a congenital bilateral atresia of the external auditory canal, and inadequate audition gain with traditional bone headband.

As much the bibliographical overhaul as the clinical case allows to assert that Bonebridge ${ }^{\circledR}$ corresponds to an alternative of treatment in the atresia of the external auditory canal and with certain benefits by on the other devices, as the surgery.

Key words: External auditory canal atresia, Bonebrigde ${ }^{\circledR}$.

\section{INTRODUCCIÓN}

La atresia del conducto auditivo externo corresponde a una patología que afecta a 1 de cada 10.0000 cada 20.000 nacidos vivos, siendo de éstos $70 \%$ unilateral, con afectación más frecuente en hombres y derecha; tan sólo $30 \%$ es bilateral'. El manejo quirúrgico sigue siendo un tanto controversial sobre todo en aquellos casos con atresia unilateral y suele reservarse hasta que los niños son capaces de tener consentimiento sobre su cirugía. No obstante existen recomendaciones sobre la realización de la cirugía, algunos en edad, otros incluso diseñaron una escala de predicción de resultados auditivos como Jahrsdoefer (1992) 2,3.

En el último tiempo gracias al desarrollo importante en los dispositivos implantables, se ha posicionado como una opción terapéutica la utilización de dispositivos de conducción ósea osteointegrados dentro de los cuales el más utilizado es el de nombre BAHA, los cuales se encuentran aprobados por la FDA, con complicaciones en baja frecuencia a pesar de muchas veces requerir dos procedimientos quirúrgicos para su colocación. El gran posicionamiento de estos dispositivos se debe a que la cirugía tiene alrededor del $93 \%$ de requerimiento de algún tipo de amplificación auditiva. Además de lo anterior resulta en dos quintos del precio que cuesta en promedio la cirugía, debido a la alta tasa de complicaciones y reintervenciones requeridas ${ }^{4}$.

Con respecto a las intervenciones quirúrgicas, ha quedado esclarecido luego de varios estudios que la mejor alternativa terapéutica, corresponde al abordaje transmastoideo, el cual tiene mejores resultados auditivos y menos aparición de reestenosis del conducto ${ }^{5}$. La cirugía a pesar de tener un resultado estético importante y significativo para los pacientes, es significativamente mayor la ganancia auditiva con dispositivos auditivos con conducción ósea, con un promedio de aproximadamente $40 \mathrm{~dB}$ en comparación con los $3 \mathrm{~dB}$ que logra la cirugía. A pesar de lo anterior, considerar la cirugía como un método terapéutico es bastante aceptado. Una cantidad importante de casos de atresia del CAE se asocian a malformaciones del pabellón auricular ${ }^{6}$. No obstante la poca ganancia auditiva que tiene la cirugía, sí tiene la ventaja de permitir la implementación de un audífono en estos pacientes.

En el último tiempo los dispositivos auditivos de conducción ósea se han transformado en el método de elección en el manejo de las hipoacusias de tipo conductivas o mixtas unilaterales que no han tenido respuesta al manejo con dispositivos tradicionales de audición. Teniendo una casuística que supera los 100.000 casos en todo el mundo, el dispositivo BAHA ha demostrado muy buenos resultados a pesar de tener algunas limitaciones importantes derivadas del diseño del implante: requiere un manejo a largo plazo de la herida operatoria para evitar complicaciones; son frecuentes las infecciones que requieren de tratamiento médico; tiene una retroalimentación que limita la ganancia máxima del dispositivo; puede ocurrir la extrusión espontánea del tornillo que usa el implante, pu- 
diendo requerir el reimplante de este mismo; finalmente existe estigmatización de los pacientes ${ }^{7}$.

En respuesta a este tipo de problemas que presentan los dispositivos auditivos con conducción ósea osteointegrados como el BAHA, se ha diseñado un nuevo dispositivo que es parcialmente implantable, que tiene una masa flotante que corresponde al transductor, que se implanta mediante 2 tornillos. Para comparar este mismo con el gold standard se utilizó una medición de la aceleración a la cual se ve sometida la cóclea, mediante un láser que mide la vibración de esta misma. Los estudios demostraron una diferencia significativa entre los dispositivos, pero solo en frecuencias mayores de $4.000 \mathrm{~Hz}$ siendo menos la transmisión del sonido para el dispositivo Bonebridge ${ }^{\circledR}$ que en el BAHA, no obstante esto ocurre en frecuencias que no tienen tanta relevancia del punto de vista funcional especialmente con el discurso hablado. Además de lo anterior se pudo evidenciar que la ganancia es discretamente menor para el dispositivo Bonebridge, siendo alrededor de $7 \mathrm{a} 10 \mathrm{~dB}$ de diferencia ${ }^{8}$.

El mayor beneficio que otorga este nuevo dispositivo es que elimina por completo los inconvenientes en la piel que presentan los dispositivos osteointegrados como el BAHA, excepto el de ex-

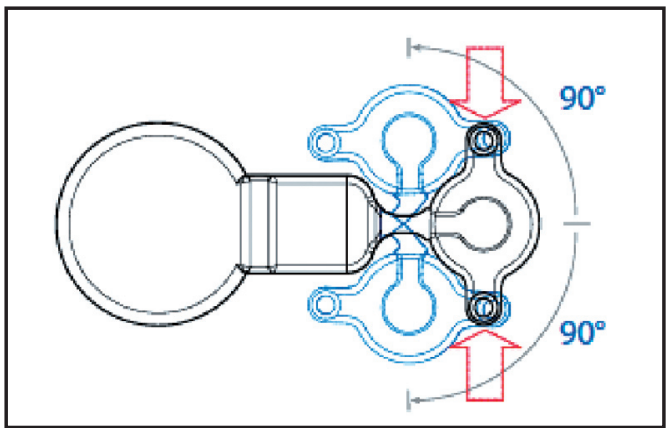

Figura $1^{10}$. Rango de movimiento horizontal.

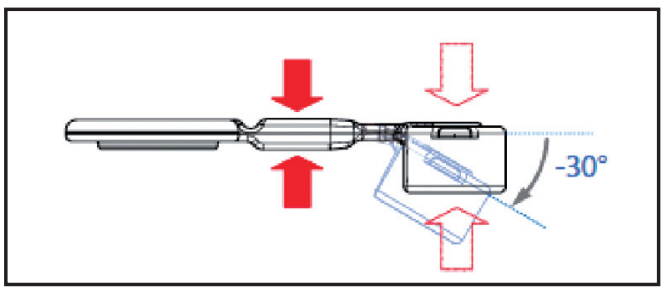

Figura $2^{10}$. Rango de movimiento vertical. trusión que podría estar presente pero con menos frecuencia del punto de vista teórico, debido al doble anclaje que tiene este dispositivo.

Uno de los temas que no se puede dejar de lado es que al ser transcutánea la estimulación se pierde un poco de ganancia, pero a su vez se gana en otros aspectos, como por ejemplo que está expuesto a menor retroalimentación y es menos susceptible a la captación de ondas radiales que pudiesen intervenir y así lo demuestra Taghavi (2012) en cadáveres9.

En relación a la atenuación transcraneal Huber (2013) demuestra una diferencia significativa con el dispositivo BAHA, siendo mayor en el caso de Bonebridge ${ }^{\circledR}$, el cual pudiese ser beneficioso en caso de hipoacusias de conducción unilaterales al disminuir la estimulación del oído sano contralateral y evitar perturbaciones de la audición de ese oído.

Debido a que no existe aún publicaciones respecto a la experiencia con este tipo de implantes en nuestro medio, se presenta el siguiente caso clínico de colocación de un dispositivo auditivo de conducción ósea transcutánea llamado Bonebridge ${ }^{\circledR}$.

\section{IMPLANTE BONEBRIDGE ${ }^{\circledR}$}

Las indicaciones auditivas habituales corresponden a pérdidas auditivas conductivas 0 mixtas con umbrales de conducción ósea igual o mayor de $45 \mathrm{~dB}$ en $0,5,1,2$ y $3 \mathrm{KHz}$. Además se incluye la hipoacusia unilateral neurosensorial grave a profunda con el contralateral en audición normal.

El dispositivo Bonebridge ${ }^{\circledR}$ corresponde a una prótesis auditiva activa implantable y de transmisión directa donde el $\mathrm{BCl}$ (implante de conducción ósea), que se coloca sobre la mastoides, está rodeado de una bobina receptora y un imán central.

El dispositivo cuenta además con un demodulador y luego una transición hasta el transductor la cual es flexible, permitiendo variaciones en la orientación del $\mathrm{BCI}$ con respecto al transductor, la transición es posible de mover en \pm 90 grados en la orientación horizontal y -30 grados en vertical, si se sobrepasa esos ángulos, se corre el riesgo de daño del dispositivo, por sobreestiramiento de los cables (Figuras 1, 2).

$\mathrm{El} \mathrm{BCl}$ es activado para su utilización una vez que se coloca el procesador auditivo externo sobre el imán de este mismo. La señal y potencia 
necesarias para poner en marcha el BC-FMT, son transmitidas por medio de un enlace inductivo de la bobina interna y procesada por medio del demodulador hacia el BC-FMT.

Es importante tener presente que el dispositivo no tiene contraindicación para la realización de radiografía, así como tampoco de tomografías computarizadas ni radioterapias. La evaluación con resonancia nuclear magnética está restringida hasta 1,5 Tesla, intensidades mayores pueden dañar el dispositivo. Finalmente no puede utilizarse electrobisturí monopolar en zonas cercanas al implante, por riesgo de daño al dispositivo ${ }^{10}$.

Figuras $3,4^{10}$. Extraídas del manual del usuario del kit de implante Bonebridge ${ }^{\circledR}$. La Figura 3 muestra el contenido del kit, el cual viene esterilizado e incluye el dispositivo, con la fresa y plantilla para su colocación en la mastoides. La Figura 4 muestra las dimensiones útiles de tener en consideración a la hora de realizar la colocación del dispositivo.

\section{CASO CLÍNICO}

Se presenta el caso de un paciente de sexo masculino, de 18 años de edad, sin antecedentes

Contenido del kit de implante (bandeja esterilizada):

1. Coil-Sizer (plantilla que representa la sección de la bobina, consulte la Figura 2, Ilamada "C-Sizer".

2. Transducer-Sizer (plantilla que representa la sección del transductor, llamada "T-Sizer")

3. Tornillos corticales (2 tornillos comunes, 1 tornillo de emergencia).

4. Fresa con tope (requiere pieza de mano con conexión dental).

5. Implante de conducción ósea (BCI 601).

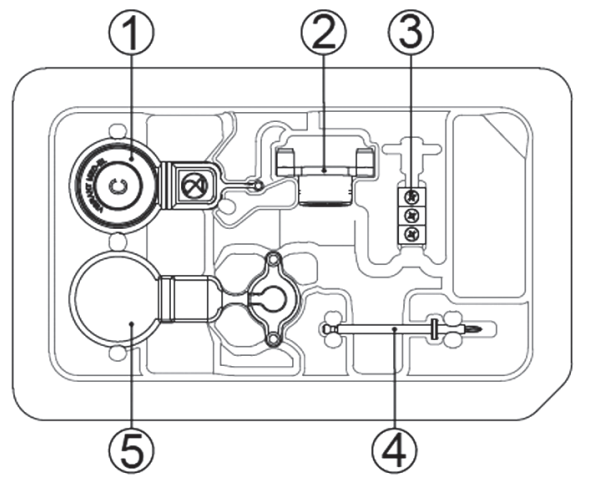

Figura 3. mórbidos de importancia y con el diagnóstico de atresia bilateral del conducto auditivo externo, con una malformación mayor del oído izquierdo asociada. Consistente en una hipoplasia de la caja timpánica, con una malformación de cadena osicular y un hueso mastoides pequeño. En el oído derecho presentaba una placa atrésica con caja timpánica normal y hueso mastoides amplio. El motivo de consulta es una hipoacusia de conducción máxima bilateral de larga data. Es usuario de un cintillo óseo tradicional, apoyado en la mastoides del oído derecho en muy mal estado y asociado a un audífono clásico PCPP antiguo en el oído derecho, aparentemente ambos con mala ganancia, según lo que relata el paciente. Debido a esto se decide la colocación de un dispositivo auditivo de conducción ósea de tipo Bonebridge ${ }^{\circledR}$ con estimulación transcutánea.

A continuación se muestra el resultado en la audiometría logrado por el implante, Figuras 5 y 6 .

\section{DISCUSIÓN}

El dispositivo Bonebridge ${ }^{\circledR}$ a pesar de ser un dispositivo con los beneficios planteados previamente tiene la limitante de la edad a la cual se puede colocar, esto debido a que requiere de una mastoides lo suficientemente desarrollada como para la colocación del transductor, el cual tiene una profundidad de $8,7 \mathrm{~mm}$. Diferentes opiniones de profesionales concuerdan que una edad segura de colocación es en mayores de 12 años, lo cual

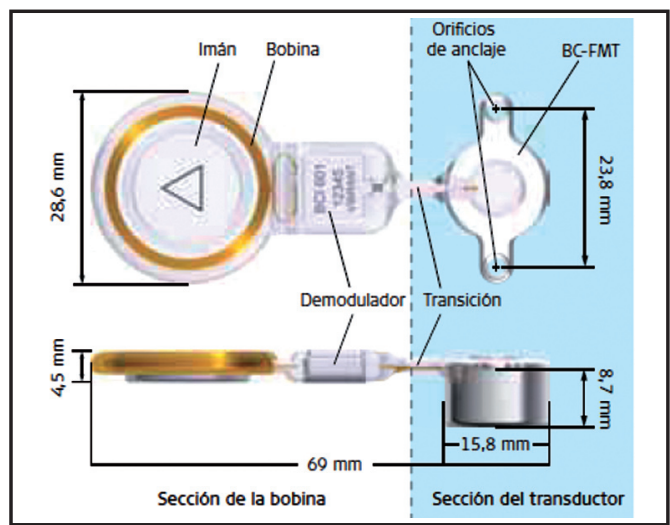

Figura 4. 


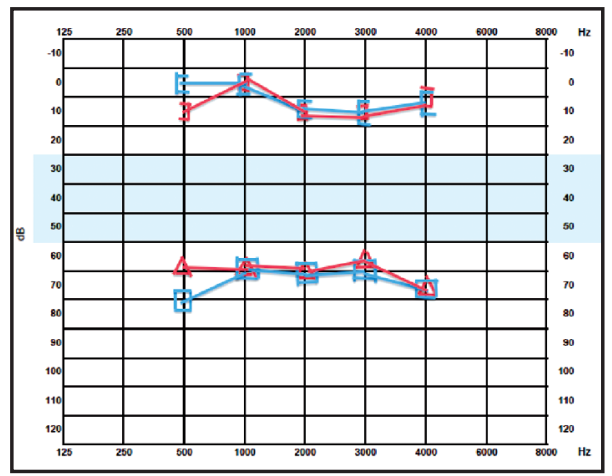

Figura 5. Esquema de audiometría preimplante.

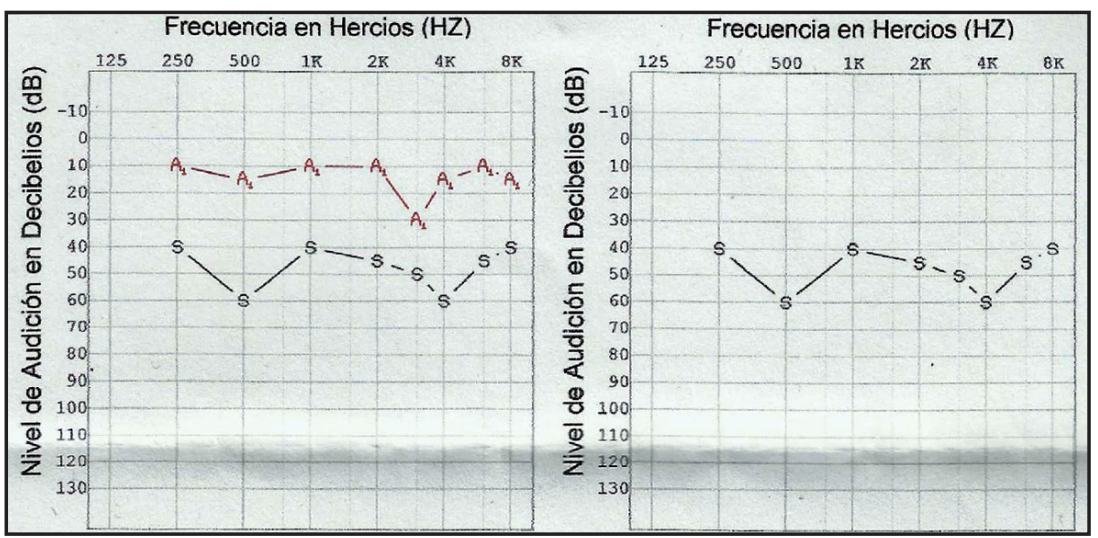

Figura 6. Ganancia auditiva lograda con el implante.

obliga a tener que esperar hasta esa edad para el manejo de la patología, obligando a buscar otras alternativas de tratamiento hasta alcanzar una edad suficiente para considerarlo como una terapia adecuada.

Existen algunos beneficios derivados del diseño que surgen como ideas interesantes de usar, como por ejemplo la colocación en cirugías radicales, colocando el FMT en la cavidad. De la misma manera es posible manejar otras hipoacusias de conducción como en el caso de una cirugía de otoesclerosis fallida o de una miringoplastía fallida. No obstante estas aplicaciones requieren de mayores estudios al respecto, antes de poder hacer recomendaciones sobre su uso y las posibles indicaciones en este tipo de patologías.

\section{BIBLIOGRAFÍA}

1. BW Jafek, GT Nager, J Strife et al. Congenital aural atresia: an analysis of 311 cases. Trans Am Acad Opthalmol Otolaryngol 1975; 80: 588-95.

2. TP Murphy, F Burstein, S Cohen. Management of congenital atresia of the external auditory canal. Otolaryngology - Head and Neck Surgery 1997; 116(6): 580-4.

3. RA Jahrsdoerfer, JW Yeakley, EA Agullar et al. Grading system for the selection of patients with congenital aural atresia. Amer J Otol 1992; 13: 6-12.

4. Adele Karen Evans, Ken Kazahaya. Canal atresia'Surgery or implantable hearing devices? The expert's question is revisited. International Journal of Pediatric Otorhinolaryngology 2007; 71: 367-74. 
5. MA El-Begermy, OI Mansour, AM El-Makhzangy, TS El-Gindy Congenital auditory meatal atresia: a numerical review. Eur Arch Otorhinolaryngol 2009; 266: 501-6.

6. Sarah Bouhabel, PierreArcand, Issam Saliba. Congenital aural atresia: Bone-anchored hearing aid vs. external auditory canal reconstruction. International Journal of Pediatric Otorhinolaryngology 2012; 76(2): 272-7.

7. CAJ Dun, HT Faber, MJ de Wolf, CW Cremers, MK HoL. An overview of different systems: the boneanchored hearing aid. Adv Otorhinolaryngol 2011; 71: 22-31.
8. AM Huber, JH Sim, YZ Xie, M Chatzimichalis, 0 UlLRICH, C RöösLI. The Bonebridge: Preclinical evaluation of a new transcutaneously-activated bone anchored hearing device. Hearing Research 2013; 301: 93-9.

9. H Taghavi, B Håkansson, S Reinfeldt, M EegOlofsSon, S AkHSHIJAN. Feedback analysis in percutaneous bone-conduction device and bone-conduction implant on a dry cranium. Otol Neurotol 2012; 33(3): 41320.

10. Manual del Usuario Bonebridge ${ }^{\mathrm{TM}}$. $\mathrm{BCl}^{\mathrm{TM}}$ Bone Conduction Implant Kit.

Dirección: David Sanhueza Costa 